\title{
Development of a prognostic index for 90-day mortality in patients discharged after admission to hospital for community-acquired pneumonia
}

\author{
A Capelastegui, ${ }^{1}$ P P España, ${ }^{1}$ J M Quintana, ${ }^{2}$ A Bilbao, ${ }^{3}$ R Menendez, ${ }^{4}$ R Zalacain, ${ }^{5}$ \\ A Torres ${ }^{6}$
}

- Additional details are published online only at http:// thorax.bmj.com/content/vol64/ issue6

${ }^{1}$ Pneumology Service, Hospital Galdakao, Galdakao, Bizkaia,

Spain; ${ }^{2}$ Research Unit, Hospital Galdakao - CIBERESP, Galdakao, Bizkaia, Spain; ${ }^{3}$ Basque

Foundation for Health Innovation and Research (BIOEF) -

CIBERESP, Sondika, Bizkaia, Spain; ${ }^{4}$ Pneumology Service, Hospital de La Fe - CIBERES 06/ 06/0028, Valencia, Spain;

${ }^{5}$ Pneumology Service, Hospital de Cruces, Baracaldo, Bizkaia, Spain; ${ }^{6}$ Pneumology Service, Hospital Clinic - CIBERES 06/06/ 0028, Barcelona, Spain

Correspondence to:

Dr A Capelastegui, Service of Pneumology, Hospital Galdakao, 48960 Galdakao, Bizkaia, Spain: alberto.capelasteguisaiz@ osakidetza.net

Received 10 March 2008 Accepted 21 January 2009 Published Online First 22 February 2009

\section{ABSTRACT}

Background: Although patients admitted to hospital for community-acquired pneumonia (CAP) experience substantial short-term mortality following hospital discharge, few studies have focused on identifying factors that predict mortality after admission to hospital in this population. The objective of this study was to develop and validate a prognostic index for 90-day mortality after hospital discharge among patients with CAP.

Methods: The prognostic index was derived in 1117 adult patients discharged between 2003 and 2007 from a general hospital following admission for CAP. It was validated in 646 consecutive patients with CAP discharged from three other hospitals between 1 November 2005 and 31 July 2006. Risk factors evaluated included host-related factors, severity upon admission, in-hospital management and bacteriology.

Results: In the derivation cohort, three factors were independently associated with 90-day mortality: preillness functional status, Charlson index (composite measure of co-morbid illnesses) and severity on admission. Mortality at 90 days was $0.7 \%$ in the low-risk group, $3.5 \%$ in the intermediate-risk group and $17.2 \%$ in the highrisk group. In the validation cohort, 90-day mortality in the three groups was $0.6 \%, 3.9 \%$ and $19.6 \%$, respectively. Compared with the low-risk group, the odds ratio for mortality was 43.5 for the high-risk group. The risk categories showed an area under the receiver operating characteristic curve of 0.79 in the derivation cohort and 0.82 in the validation cohort.

Conclusions: The prognostic index accurately stratifies patients admitted to hospital for CAP into low-, intermediate- and high-risk groups for 90-day mortality on discharge. The use of this index could help clinicians improve outcomes in this vulnerable population by targeting specific interventions to each group.

With more than 5 million cases occurring annually in the USA alone, community-acquired pneumonia (CAP) is a leading cause of hospital admissions as well as morbidity and mortality. Numerous investigations have yielded important information about factors that influence the evolution and treatment of CAP during hospitalisation..$^{1-5}$ The period after discharge from hospital, though a promising step in the resolution of CAP, should not be considered an all-clear signal. On the contrary, patients who survive admission to hospital for CAP are prone to readmission and are at high risk of dying. ${ }^{6-9}$ The 1-year mortality is considerably higher than that of the general population or a control population admitted to hospital for reasons other than CAP. ${ }^{8}$ To date, only a few trials have examined risk factors for shortterm mortality focusing on the post-discharge period for a CAP-related hospital admission. ${ }^{67}$

A study conducted in North America identified clinical factors that were useful in deciding whether a patient with CAP was sufficiently stable to be discharged from hospital. ${ }^{6}$ The presence of two or more features of clinical instability predicted a significant chance of readmission or mortality. This assessment is now recommended as part of discharge planning. ${ }^{10}$ However, a patient's trajectory after discharge is influenced by numerous other factors including host factors, bacteriological factors, the severity of CAP on admission, the evolution of the disease during hospitalisation and the treatment received. ${ }^{11}$

The aim of this study was to design and validate an accurate easy-to-use index, the CAP-90, capable of classifying adults with CAP as low, intermediate or high risk for 90-day mortality after hospital discharge.

\section{METHODS}

\section{Selection criteria}

CAP was defined as a pulmonary infiltrate on the chest radiograph not known to be old and symptoms consistent with pneumonia including cough, dyspnoea, fever and/or pleuritic chest pain not acquired in a hospital or a nursing home. Patients with pneumonia were excluded if they were known to be positive for HIV, chronically immunosuppressed (defined as immunosuppression for solid organ transplantation, post-splenectomy, receiving $\geqslant 10 \mathrm{mg} /$ day prednisone or equivalent for more than 30 days, treatment with other immunosuppressive agents or neutropenia $\left(<1.0 \times 10^{9} / 1\right.$ neutrophils $\left.)\right)$ or who had been hospitalised for the previous 14 days. The study sample was restricted to patients who survived the index hospitalisation.

\section{Derivation and validation cohort}

The prognostic index was derived in a cohort from Galdakao Hospital, a 400-bed general teaching hospital in the Basque Country (northern Spain) serving a population of 300000 inhabitants. A total of 1189 consecutive patients aged $\geqslant 18$ years admitted to Galdakao Hospital for CAP between 15 July 2003 and 30 June 2007 were prospectively enrolled in an observational cohort study. Of these, 1117 survived the index 
Table 1 Characteristics of patients admitted to hospital with community-acquired pneumonia who survived to hospital discharge in the derivation and validation cohorts

\begin{tabular}{|c|c|c|c|}
\hline Characteristics & $\begin{array}{l}\text { Derivation cohort } \\
(\mathrm{n}=1117)\end{array}$ & $\begin{array}{l}\text { Validation cohort } \\
\text { ( } \mathrm{n}=646 \text { ) }\end{array}$ & p Value \\
\hline \multicolumn{4}{|l|}{ Host-related } \\
\hline Mean (SD) baseline functional status* & $22.9(11.0)$ & $19.8(9)$ & $<0.001$ \\
\hline Baseline functional status* & & & $<0.001$ \\
\hline 15 & $440(42.5)$ & $391(63.1)$ & \\
\hline $16-30$ & $372(35.9)$ & $160(25.8)$ & \\
\hline$>30$ & $224(21.6)$ & $69(11.1)$ & \\
\hline Mean (SD) age (years) & $69.4(16.6)$ & $66.9(16.8)$ & 0.003 \\
\hline Age $>75$ years & $488(43.7)$ & $244(37.8)$ & 0.02 \\
\hline Women & $388(34.7)$ & $222(34.4)$ & 0.88 \\
\hline Charlson index & & & 0.008 \\
\hline 0 & $389(34.9)$ & $237(36.8)$ & \\
\hline 1 & $367(32.9)$ & $178(27.6)$ & \\
\hline 2 & $214(19.2)$ & $113(17.6)$ & \\
\hline$\geqslant 3$ & $144(12.9)$ & $116(18)$ & \\
\hline \multicolumn{4}{|l|}{ Severity of illness on admission } \\
\hline PSI risk class $\dagger$ & & & 0.07 \\
\hline I-III & $574(51.4)$ & $359(55.8)$ & \\
\hline IV-V & $543(48.6)$ & $284(44.2)$ & \\
\hline CURB scoret. & & & 0.11 \\
\hline 0 & $403(36.1)$ & $214(33.1)$ & \\
\hline 1 & $460(41.2)$ & $257(39.8)$ & \\
\hline$>1$ & $254(22.7)$ & $175(27.1)$ & \\
\hline Bilateral or multilobar radiographic involvement & $264(23.6)$ & $144(22.4)$ & 0.54 \\
\hline \multicolumn{4}{|l|}{ Process of care } \\
\hline Appropriate antibiotic & $1040(93.2)$ & $527(86.5)$ & $<0.001$ \\
\hline Antibiotics within $8 \mathrm{~h}$ of arrival from ED & $955(87.7)$ & $538(86.1)$ & 0.34 \\
\hline Antibiotics prior to admission & $247(22.1)$ & $151(23.7)$ & 0.43 \\
\hline Mechanical ventilation & $16(1.4)$ & $15(2.3)$ & 0.16 \\
\hline \multicolumn{4}{|l|}{ Length of stay (days) } \\
\hline Mean (SD) & $4.2(3.8)$ & $9.1(8.1)$ & $<0.001$ \\
\hline$>3$ & $510(45.7)$ & $591(91.6)$ & $<0.001$ \\
\hline \multicolumn{4}{|l|}{ In-hospital evolution } \\
\hline Treatment failure & $116(10.4)$ & $56(8.7)$ & 0.25 \\
\hline Septic shock & $35(3.1)$ & $24(3.7)$ & 0.51 \\
\hline Number of instability criteria on discharge & & & $<0.001$ \\
\hline 0 & $684(63.2)$ & $615(95.9)$ & \\
\hline$\geqslant 1$ & $399(36.8)$ & $26(4.1)$ & \\
\hline Causal microorganism & & & $<0.001$ \\
\hline Streptococcus pneumoniae & $250(22.4)$ & $124(19.2)$ & \\
\hline Atypical bacterial pathogens & $114(10.2)$ & $14(2.2)$ & \\
\hline Mixed infections & $100(9.0)$ & $9(1.4)$ & \\
\hline Legionella pneumophila & $38(3.4)$ & $22(3.4)$ & \\
\hline Atypical virus pathogens & $13(1.2)$ & $3(0.5)$ & \\
\hline Others & $37(3.3)$ & $40(6.2)$ & \\
\hline Unknown & $565(50.9)$ & $434(67.2)$ & \\
\hline
\end{tabular}

Data are presented as numbers (percentage) unless otherwise stated. Percentages exclude patients with missing data. Treatment failure and instability criteria on discharge are defined in the text.

*Baseline functional status was measured by Katz index ( $n=1036$ in derivation cohort and 620 in validation cohort); functional status range 15-52 (excellent functional status, 15).

$\dagger$ Severity of illness on admission assessed with PSI (Pneumonia Severity Index).

¥ Severity of illness on admission assessed with CURB score (Confusion, Urea nitrogen, Respiratory rate, Blood pressure).

Additional details are provided in the online data supplement (tables E2, E3 and E4).

ED, emergency department; PSI, Pneumonia Severity Index; SD, standard deviation.

hospitalisation and satisfied all the criteria for inclusion in the study. An external validation cohort was formed with 671 consecutive adult patients hospitalised for CAP between 1 November 2005 and 31 July 2006 in three nearby large teaching hospitals (Cruces Hospital in Vizcaya, Clinic Hospital in Barcelona and La Fe Hospital in Valencia). Of these, 646 survived the index hospitalisation and satisfied all the criteria for inclusion in the analysis.

\section{Predictors of mortality}

Variables that may predict short-term mortality among patients discharged following admission to hospital for CAP were selected and defined after an exhaustive literature review. To promote clinical utility, we arranged these into host-related factors (functional status measured by Katz index ${ }^{12}$ age, gender, co-morbid illness measured by Charlson index $\left.{ }^{13}\right)$, severity of illness upon admission (CURB (Confusion, Urea nitrogen, 
Table 2 Factors significantly associated in univariate analyses with mortality within 90 days of hospital discharge in the derivation cohort $(\mathrm{n}=1117)$

\begin{tabular}{|c|c|c|}
\hline Factors & OR $(95 \% \mathrm{CI})$ & p Value \\
\hline \multicolumn{3}{|l|}{ Host-related } \\
\hline Baseline functional status (continuous) ${ }^{*}$ & 1.09 (1.07 to 1.11$)$ & $<0.001$ \\
\hline \multicolumn{3}{|l|}{ Baseline functional status* } \\
\hline $16-30$ vs 15 & $4.86(1.36$ to 17.34$)$ & 0.02 \\
\hline$>30$ vs 15 & $22.53(6.79$ to 74.70$)$ & $<0.001$ \\
\hline Age (years) & $1.06(1.04$ to 1.09$)$ & $<0.001$ \\
\hline Age $>75$ years & $3.33(1.84$ to 6.03$)$ & $<0.001$ \\
\hline Male vs female & 1.96 (1.02 to 3.77$)$ & 0.04 \\
\hline \multicolumn{3}{|l|}{ Charlson index } \\
\hline 1 vs 0 & 3.05 (1.09 to 8.54$)$ & 0.03 \\
\hline 2 vs 0 & $6.21(2.24$ to 17.19$)$ & $<0.001$ \\
\hline$\geqslant 3$ vs 0 & 12.39 (4.55 to 33.69$)$ & $<0.001$ \\
\hline \multicolumn{3}{|l|}{ Severity of illness on admission } \\
\hline \multicolumn{3}{|l|}{ CURB score $\dagger$} \\
\hline 1 vs 0 & $1.97(0.92$ to 4.22$)$ & 0.08 \\
\hline$>1$ vs 0 & 3.91 (1.83 to 8.37$)$ & $<0.001$ \\
\hline $\begin{array}{l}\text { Bilateral or multilobar radiographic } \\
\text { involvement }\end{array}$ & $1.61(0.90$ to 2.89$)$ & 0.11 \\
\hline \multicolumn{3}{|l|}{ Process of care } \\
\hline Appropriate antibiotic & $0.93(0.33$ to 2.64$)$ & 0.89 \\
\hline Antibiotics within $8 \mathrm{~h}$ & $0.82(0.38$ to 1.77$)$ & 0.60 \\
\hline Antibiotics prior to admission & $1.22(0.65$ to 2.27$)$ & 0.54 \\
\hline Mechanical ventilation $\$$ & - & - \\
\hline \multicolumn{3}{|l|}{ Length of stay (days) } \\
\hline Length of stay (days) & $1.05(1.00$ to 1.10$)$ & 0.06 \\
\hline$>3$ vs $\leqslant 3$ & $1.70(0.98$ to 2.94$)$ & 0.06 \\
\hline \multicolumn{3}{|l|}{ In-hospital evolution } \\
\hline Treatment failure & $2(0.98$ to 4.09$)$ & 0.06 \\
\hline Septic shock & 0.56 (0.08 to 4.17$)$ & 0.57 \\
\hline \multicolumn{3}{|l|}{ Number of instability criteria on discharge } \\
\hline$\geqslant 1$ vs 0 & $1.70(0.99$ to 2.93$)$ & 0.06 \\
\hline \multicolumn{3}{|l|}{ Causal microorganism§ } \\
\hline Streptococcus pneumoniae & Reference & \\
\hline Atypical bacterial or virus pathogens & $0.48(0.13$ to 1.73$)$ & 0.26 \\
\hline Mixed infections & $0.20(0.03$ to 1.56$)$ & 0.13 \\
\hline Others & $1.75(0.47$ to 6.52$)$ & 0.40 \\
\hline Unknown & 1.35 (0.69 to 2.64$)$ & 0.38 \\
\hline
\end{tabular}

Treatment failure and instability criteria on discharge are defined in text. Each factor was examined individually.

*Baseline functional status was measured by the Katz index ( $n=1036)$; functional status range 15-52 (excellent functional status, 15). Baseline functional status was analysed as continuous and categorical variable separately.

$\dagger$ Severity of illness on admission was assessed with CURB score (Confusion, Urea nitrogen, Respiratory rate, Blood pressure).

\#No deaths within 90 days of discharge were recorded for patients with mechanical ventilation during hospitalisation.

§ln the univariate analyses for causal microorganisms, the Streptococcus pneumoniae category was considered as the reference group.

OR, odds ratio; $95 \% \mathrm{Cl}, 95 \%$ confidence interval.

Respiratory rate, Blood pressure) score,,$^{14}$ bilateral or multilobar radiographic involvement), factors related to in-hospital evolution (treatment failure, septic shock), stability upon hospital discharge, treatment-related factors (appropriate antibiotic according to local practice guidelines that are similar to ATS guidelines, ${ }^{15}$ administration of antibiotics within $8 \mathrm{~h}$ of admission, antibiotics taken prior to admission and use of mechanical ventilation), length of stay and bacteriology-related factors.

Additional details are provided in the online data supplement.

\section{Outcomes}

The primary outcome was all-cause mortality within 90 days of the index hospital discharge date. Vital status was initially determined by telephone interview 90 days after discharge. Reported deaths and dates of deaths were confirmed by review of medical records, public death registries, or both. Mortality data were available for the entire cohort within 90 days, except for two cases in the validation sample for whom the exact dates of death were unknown.

\section{Statistical analysis}

Descriptive statistics included frequency tables and mean and standard deviation. $\chi^{2}$ and Fisher exact tests were performed for categorical variables, and the Student $t$ test and non-parametric Wilcoxon tests were performed for continuous variables. Mortality within 90 days of hospital discharge was also compared between patients in the derivation and validation cohorts by means of a logistic regression model.

Univariate logistic regression models were first used to identify risk factors associated with 90-day mortality; we determined the odds ratio (OR) and 95\% confidence interval ( $95 \%$ CI). Multivariate logistic regression models were then performed to identify the statistical significance and weight of each risk factor. The dependent variable was mortality within 90 days of discharge; the independent variables were factors identified as having $\mathrm{p}<0.15$ in the univariate analysis. Two multivariate models were considered: one included baseline functional status, as measured by the Katz index; the other did not. The predictive accuracy of the prognostic models was determined by calculating the area under the receiver operating characteristic (ROC) curve (discrimination) ${ }^{16}$ and by comparing predicted and observed mortality by means of the Hosmer and Lemeshow test (calibration). ${ }^{17}$ Area under the curve (AUC) values were compared using the non-parametric method described by Hanley and NcNeil. ${ }^{18}$ Points were assigned to each predictive variable from the $\beta$ parameter obtained in the 90-day mortality multivariate model. To create the CAP-90 index, we added the points assigned to each of the selected variables, with a higher score corresponding to a higher likelihood of 90-day mortality. Once the index was developed, we created three categories (low, intermediate and high risk) in relation to the predicted mortality.

After developing the CAP-90 index, we attempted to validate it in a separate cohort. First, the predictive accuracy of the point-based CAP-90 index was determined by calculating the AUC values in each cohort. Second, logistic regression models were performed in each cohort, considering the low-risk group as the reference category. Finally, Kaplan-Meier curves were constructed for each of the three risk groups and comparisons were performed by the log-rank test. For the comparison of survival curves between derivation and validation cohorts, a Cox proportional hazard regression model was created, considering the risk group, the cohort and the interaction between risk group and cohort as independent variables.

All effects were considered significant at $p<0.05$. All statistical analyses were performed using SAS for Windows statistical software Version 8.0 (SAS Institute, Carey, North Carolina, USA) and S-Plus 2000 (MathSoft, Seattle, USA, 1999).

\section{RESULTS}

Patients in the derivation and validation cohorts differed significantly in several key characteristics (table 1). In the derivation cohort, $36.8 \%$ of patients were discharged with one or more instability criteria compared with $4.1 \%$ in the validation cohort. However, the rate of 90-day mortality after discharge was $4.9 \%$ (55/1117) in the derivation cohort and 3.3\% 
Table 3 Risk factors significantly associated in multivariate analyses with mortality within 90 days of hospital discharge in the derivation cohort $(n=1117)$

\begin{tabular}{|c|c|c|c|c|}
\hline Risk factors & $\beta$ parameter & OR $(95 \% \mathrm{CI})$ & p Value & Points \\
\hline Intercept & -5.33 & & & \\
\hline \multicolumn{5}{|l|}{ Baseline functional status* } \\
\hline $16-30$ vs 15 & 1.26 & $3.51(0.96$ to 12.79$)$ & 0.06 & 3 \\
\hline$>30$ vs 15 & 2.61 & 13.62 (3.96 to 46.85$)$ & $<0.001$ & 6 \\
\hline \multicolumn{5}{|l|}{ Charlson index } \\
\hline$>1$ vs $0+1$ & 0.87 & $2.38(1.24$ to 4.59$)$ & 0.009 & 2 \\
\hline \multicolumn{5}{|l|}{ CURB score $\dagger$} \\
\hline$>1$ vs $0+1$ & 0.79 & $2.20(1.16$ to 4.15$)$ & 0.01 & 2 \\
\hline AUC & & 0.81 & & \\
\hline Hosmer-Lemeshow $\mathrm{p}$ value: & & 0.910 & & \\
\hline
\end{tabular}

\footnotetext{
All risk factors were examined jointly.

$\mathrm{n}=1033$ (45 deaths, 988 survivors). No answers were received to Katz index for 84 cases (10 deaths).

* Baseline functional status was measured by the Katz index $(n=1036)$; functional status range $15-52$ (excellent functional status, 15).

$\uparrow$ Severity of illness on admission was assessed with the CURB score (Confusion, Urea nitrogen, Respiratory rate, Blood pressure).

fA significant value for the Hosmer-Lemeshow statistic indicates a significant deviation between predicted and observed outcomes.

AUC, area under the receiver operating characteristic curve; $\mathrm{OR}$, odds ratio; $95 \% \mathrm{Cl}, 95 \%$ confidence interval; $\beta$ parameter, estimated $\beta$ coefficient.
}

$(21 / 646)$ in the validation cohort, a non-significant difference that remained non-significant after adjustment. The introduced interaction terms were not statistically significant. The comorbidities of patients in the derivation and validation cohorts are described in table E1 in the online supplement.

\section{Derivation of prognostic index}

In univariate analyses, several host-related factors, severity of illness on admission and clinical instability at the time of discharge were associated with the likelihood of 90-day mortality (table 2).

In multivariate analyses that included the Katz index (model 1), three factors were found to be independently associated with 90-day mortality: the Katz index, Charlson index and the CURB score at admission to the emergency department (table 3). When the analyses were repeated without the Katz index (model 2), the Charlson index and CURB score remained independently associated with 90-day mortality (see table E5 available online). In addition, age emerged as a significant predictor. Both logistic models showed excellent discrimination, with AUC values of 0.81 in model 1 and 0.74 in model 2, but these were significantly different $(p=0.01)$. Both models were also well calibrated, with Hosmer-Lemeshow p values of 0.910 in model 1 and 0.919 in model 2. In model 1, if we performed the analyses using the CURB- 65 or the CRB- 65 instead of the CURB score as a marker for the severity of acute illness, the results were very similar (see tables E6 and E7 available online). In model 2, if we used as risk factor the CURB-65 score or CRB65 score instead of the CURB score and age, the discrimination of this new model was similar to model 2 (see table E8 available online).

\section{Validation of the prognostic index}

Based on the results of model 1, patients were divided into three groups from low to high risk (table 4). In the validation cohort the mortality risk ranged from $0.6 \%$ in those with $0-2$ points on the CAP-90 index to $19.6 \%$ in those with $>7$ points (trend test, $p<0.001$ ), with a 43-fold increase in the odds ratios between the referent low-risk group and the high-risk group. The risk categories showed excellent discrimination, with AUC values of 0.82 in the validation cohort. Kaplan-Meier survival curves of the three risk groups in the derivation and validation cohorts had markedly different survival trajectories with persistent differences in 90 -day mortality (fig 1 ). No significant differences were observed in the survival curves between the derivation and validation cohorts in any of the risk groups. Data based on the result of model 2 are available in the online supplement (table E9, fig E1).

\section{DISCUSSION}

This study confirmed the substantial rates of short-term mortality among patients hospitalised with CAP following discharge seen in previous studies. ${ }^{6-8}$ Equally important, we identified a simple easy-to-apply prognostic index composed of three variables-functional status before admission to hospital, the Charlson index and the severity of CAP at the time of presentation to the hospital-that can categorise patients in this population into low, intermediate and high risk for 90-day

Table 4 Validation of the CAP-90 index: 90-day mortality in the derivation and validation cohorts by index score categories

\begin{tabular}{|c|c|c|c|c|c|}
\hline \multirow[b]{2}{*}{ Risk group (points) } & \multicolumn{2}{|c|}{ Derivation cohort $(n=1117)$} & \multicolumn{2}{|c|}{ Validation cohort $(n=646)$} & \multirow[b]{2}{*}{ p Value $\dagger$} \\
\hline & $\begin{array}{l}\text { No who died/no } \\
\text { at risk }(\%)^{*}\end{array}$ & OR (95\% Cl) & $\begin{array}{l}\text { No who died/no } \\
\text { at risk }(\%)^{*}\end{array}$ & OR (95\% Cl) & \\
\hline Intermediate risk (3-7) & $16 / 454(3.5)$ & 5.18 (1.50 to 17.89$)$ & $8 / 205(3.9)$ & 7.23 (1.52 to 34.36$)$ & 0.81 \\
\hline High risk $(>7)$ & $26 / 151(17.2)$ & 29.47 (8.77 to 98.97 ) & $11 / 56(19.6)$ & 43.50 (9.34 to 202.54$)$ & 0.66 \\
\hline AUC & & 0.79 & & 0.82 & \\
\hline
\end{tabular}

The low-risk group was considered as the reference group.

${ }^{*} \mathrm{p}<0.001$ for the Cochran-Armitage test for trend. $\dagger \chi^{2}$ or Fisher exact tests for comparison of mortality proportion between the derivation and validation cohorts.

AUC, area under the receiver operating characteristic curve; $\mathrm{OR}$, odds ratio; $95 \% \mathrm{Cl}, 95 \%$ confidence interval. 

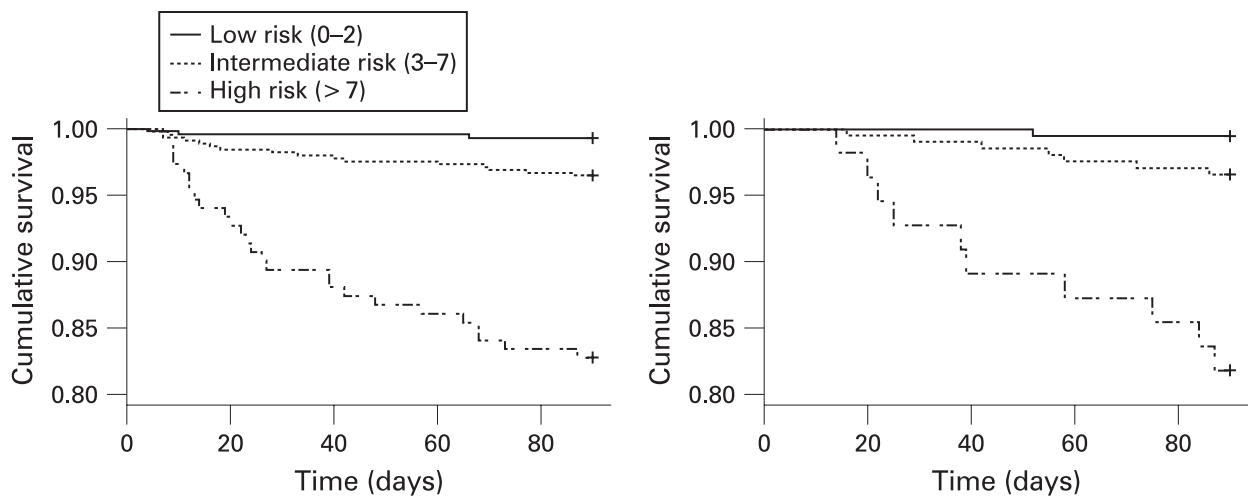

Figure 1 Kaplan-Meier survival curves for the three risk groups in the derivation (left) and validation (right) cohorts according to the CAP-90 prognostic index. Baseline functional status: 6 points for $>30$ and 3 points for $16-30$ measured by the Katz index; 2 points for Charlson index $>1$; 2 points for CURB score $>1$. The log-rank test detected statistically significant differences between all curves $(p<0.01)$ in both cohorts.

mortality. The model we developed shows excellent discrimination and calibration.

To our knowledge, this is the first study to identify predictors of short-term mortality following hospital discharge for CAP and combines them into a single score. In addition to evaluating information that is nearly always readily available at the time of admission-such as the severity of CAP, host status and parameters of clinical stability in the $24 \mathrm{~h}$ prior to dischargewe also examined the evolution of the disease during the index hospital admission, the pathogen responsible for pneumonia, process of care variables and the length of stay.

A new finding in our study is that health status before the acute illness, as measured by the Katz index, is a strong predictor of short-term mortality in patients with CAP who survive to hospital discharge. The Katz index is not routinely used to assess functional status and can be seen as a complicated tool for use in daily practice. However, the survey takes only a few minutes and can be used to satisfy the Centers for Medicare and Medicaid Services requirements for collecting functional status data. ${ }^{19}$ As an alternative, the Katz index can be replaced by variables included in a second model (age, Charlson index and the CURB score at admission) which retains a high discriminatory power. These parameters are easily obtained in daily clinical practice.

The inclusion of functional status may provide the CAP-90 with good discriminatory power. A study conducted among older patients with CAP found an association between preillness functional status and mortality risk, ${ }^{20}$ a finding previously documented only among residents of long-term care facilities. ${ }^{21}$ Pneumonia-specific scoring systems such as the Pneumonia Severity Index (PSI) ${ }^{3}$ or CURB-65 scale $e^{5}$ have proved to offer valuable prognostic information for adults with CAP. However, they do not provide an assessment of patients' functional status which, among elderly subjects, appears to be a vital contributor to outcomes such as disability and survival. ${ }^{22} 23$

Numerous studies have highlighted the importance of comorbid disease in determining patient outcomes after a critical illness, ${ }^{24}{ }^{25}$ including CAP. ${ }^{7}$ Our results show that co-morbid illness measured by the Charlson index is a major independent predictor of 90-day mortality. Co-morbid illness scales have proved useful in identifying subgroups of patients who are more likely to benefit from high-quality care. $^{26}$ Our findings emphasise the importance of optimal management of comorbidities, given evidence that changes to the organisation and delivery of care-including improving patients' self-management education, instituting programmes using education, feedback or reminders for healthcare providers, and ensuring continuity of care-can improve the quality of care and certain outcomes. $^{27} 28$

Previous studies have reported an association between CAP severity on admission and 30-day mortality. ${ }^{3}{ }^{14}$ Our data show that this association persists for at least 90 days after discharge. This raises an interesting question: Why is the extent of illness and physiological compromise on admission associated with mortality 90 days after discharge? A recent study showed that persistent inflammation at hospital discharge after CAP is associated with higher mortality over the subsequent 3 months. The authors speculate that the high inflammatory concentrations observed at hospital discharge in more than half of their patients could be due to an interaction between poor chronic health and acute illness. ${ }^{29}$ Among patients admitted with severe CAP, systemic inflammation may resolve slowly and persist long after discharge. Indeed, the levels of inflammatory cytokines induced during an episode of CAP have been correlated with the severity of pulmonary infection. ${ }^{30}$

The significant differences observed in the characteristics and management of patients in the derivation and validation cohorts are almost certainly related to the implementation of practice guidelines for CAP in Galdakao Hospital, which provided the derivation cohort, in 2000. ${ }^{31}$ The use of these guidelines may have led to higher admission rates among older patients and those with poorer functional status upon admission, as well as shorter lengths of stay. ${ }^{32}$ The substantially higher rate of patients with any instability criteria on discharge in the derivation cohort (36.8\%) than in the validation cohort $(4.1 \%)$ is associated with the shorter length of stay and the conservative threshold for temperature used to define instability $\left(\geqslant 37.2^{\circ} \mathrm{C}\right) .^{6}$ Despite the large difference in clinical instability between the cohorts, the rate of 90-day mortality after discharge was similar. Neither length of stay nor the presence of instability criteria (which were presented differently in the two cohorts) were statistically significant when studied as interaction terms in relation to mortality.

Our study has several strengths: detailed clinical prospective data collection, large sample size, use of a standardised tool for assessing pre-illness status and validation in an independent sample whose characteristics differed substantially at baseline from the derivation cohort.

The study also has some limitations. We did not identify the actual cause of death in our subjects because many patients died at home without autopsies. Although these data are potentially available from death certificates, the deficiencies in this 
approach are well documented. ${ }^{33}$ In addition, the validity of using a clinical review committee to determine cause of death for patients with CAP has not been previously established. ${ }^{7}$ We determined patients' pre-illness functional status from their responses to a questionnaire at the time of diagnosis. This could bias the results in either direction: acutely ill patients could overestimate the presence of symptoms and functional limitations before the onset of illness or underestimate these symptoms and limitations. However, previous studies have demonstrated the validity of retrospective reports for assessing functional status before admission to hospital in acutely ill patients. ${ }^{34}{ }^{35}$ Finally, one needs to be appropriately cautious in the interpretation of our study findings because of the relatively small number of post-discharge deaths.

In summary, we have developed a prognostic index (CAP-90) that uses information from just three readily available factors to estimate short-term mortality following discharge for patients admitted to hospital with CAP. This index could improve the management of CAP in several ways. By identifying low-, intermediate- and high-risk patients, it could help clinicians target specific interventions to each group; for example, a lowrisk patient (index score 0-2) could be safely discharged. Optimal management of intermediate- and high-risk patients remains to be determined. Interventions such as immunisations for influenza and Pneumococcus when indicated, review of signs or symptoms that suggest a worsening of the underlying condition, an appropriate outpatient follow-up with a physician and non-specific measures such as regular physical activity and better social support may improve post-discharge outcomes.

Physicians treating patients with CAP should be aware that discharge of patients with risk factors is common and increases the risk of a poor post-discharge outcome. Although it is possible that the natural course of some of the disease processes may not be alterable, earlier recognition of high-risk patients maximises the potential for interventions to minimise subsequent morbidity and mortality.

Funding: None.

Competing interests: None.

Ethics approval: The local ethics committees approved the project.

\section{REFERENCES}

1. España PP, Capelastegui A, Gorordo I, et al. Development and validation of a clinical prediction rule for severe community-acquired pneumonia. Am J Respir Crit Care Med 2006:174:1249-56.

2. Kaplan V, Angus DC, Griffin MF, et al. Hospitalized community-acquired pneumonia in the elderly: age -and- sex-related patterns of care and outcome in the United States. Am J Respir Crit Care Med 2002;165:766-72.

3. Fine MJ, Auble TE, Yealy DM, et al. A prediction rule to identify low-risk patients with community-acquired pneumonia. N Engl J Med 1997:336:243-50.

4. Ewig S, Ruiz M, Mensa J, et al. Severe community-acquired pneunonia: assessment of severity criteria. Am J Respir Crit Care Med 1998;158:1102-8.

5. Lim WS, Van der Eerden MM, Laing $R$, et al. Defining community-acquired pneumonia severity on presentation to hospital: an international derivation and validation study. Thorax 2003;58:377-82.

6. Halm EA, Fine MJ, Kapoor WN, et al. Instability on hospital discharge and the risk of adverse outcomes in patients with pneumonia. Arch Intern Med 2002;162:1278-84.
7. Mortensen EM, Coley CM, Singer DE, et al. Causes of death for patients with community-acquired pneumonia. Results from the pneumonia patients outcomes research team cohort study. Arch Intern Med 2002;162:1059-64.

8. Kaplan V, Clermont G, Griffin MF, et al. Pneumonia. Still the old man's friend? Arch Intern Med 2003;163:317-23.

9. Waterer GW, Kessler LA, Wunderink RG. Medium-term survival after hospitalization with community-acquired pneumopnia. Am J Respir Crit Care Med 2004;169:910-4.

10. Macfarlene JT, Boldy D. 2004 update of BTS pneumonia guidelines: what's new? Thorax 2004;59:364-6.

11. Niederman MS. Understanding the natural history of community-acquired pneumonia resolution: vital information for duration of therapy. Clin Infect Dis 2004;39:1791-3

12. Katz S, Downs TD, Cash HR, et al. Progress in development of the index of ADL. Gerontologist 1970;10:20-30.

13. Charlson M, Szatrowski TP, Peterson J, et al. Validation of a combined comorbidity index. J Clin Epidemiol 1994;47:1245-51.

14. Lim WS, Macfarlane JT, Boswell TC, et al. Study of community acquired pneumonia aetiology (SCAPA) in adults admitted to hospital: implications for management guidelines. Thorax 2001;56:296-301.

15. Niederman MS, Mandell LA, Anzueto A, et al. Guidelines for the management of adults with community-acquired pneumonia. Diagnosis, assessment of severity, antimicrobial therapy, and prevention. Am J Respir Crit Care Med 2001;163:1730-54.

16. Hanley JA, McNeil BJ. The meaning and use of the area under a receiver operating characteristic (ROC) curve. Radiology 1982:143:29-36.

17. Hosmer DW, Lemeshow S. Applied logistic regression. New York: John Wiley, 1989:140-5.

18. Hanley JA, McNeil BJ. A method of comparing areas under receiver operating characteristic curves derived from the same cases. Radiology 1983;148:839-43.

19. Inouye SK, Bogardus ST, Vitagliano G, et al. Burden of illness score for elderly persons. Risk adjustment incorporating the cumulative impact of diseases, physiologic abnormalities, and functional impairments. Med Care 2003;41:70-83.

20. Torres $\mathbf{O H}, \mathbf{M u n ̃ o z} \mathrm{J}$, Ruiz $\mathrm{D}$, et al. Outcome predictors of pneumonia in elderly patients: importance of functional assessment. J Am Geriatr Soc 2004;52:1603-9.

21. Muder RR, Brennen C, Swenson DL, et al. Pneumonia in a long-term care facility: a prospective study of outcome. Arch Intern Med 1996;156:2365-70.

22. Inouye SK, Peduzzi PN, Robison JT, et al. Importance of functional measures in predicting mortality among older hospitalized patients. JAMA 1998;279:1187-93.

23. Metlay JP, Fine MJ, Schulz R, et al. Measuring symptomatic and functional recovery in patients with community-acquired pneumonia. J Gen Intern Med 1997:12:423-30.

24. Poses RM, McClish DK, Smith WR, et al. Prediction of survival of critically ill patients by admission comorbidity. J Clin Epidemiol 1996;49:743-7.

25. de Groot V, Beckerman H, Lankhorst GJ, et al. How to measure comorbidity. A critical review of available methods. J Clin Epidemiol 2003;56:221-9.

26. Meng L, Maskarinec G, Lee J, et al. Lifestyle factors and chronic diseases: application of a composite risk index. Prev Med 1999;29:296-304.

27. Bodenheimer T, Lorig $\mathrm{K}$, Holman $\mathrm{H}$, et al. Patient self-management of chronic disease in primary care. JAMA 2002;288:2469-75.

28. Weingarten SR, Henning JM, Badamgarav E, et al. Interventions used in disease management programmes for patients with chronic illness-which ones work? Metaanalysis of published reports. BMJ 2002;325:925-8.

29. Yende S, D'Angelo G, Kellum JA, et al. Inflammatory markers at hospital discharge predict subsequent mortality after pneumonia and sepsis. Am J Respir Crit Care Med 2008; 177:1242-7

30. Glynn P, Coakley R, Kilgallen I, et al. Circulating interleukin 6 and interleukin 10 in community acquired pneumonia. Thorax 1999;54:51-5.

31. Capelastegui A, España PP, Quintana JM, et al. Improvement of process-of-care and outcomes after implementing a guideline for management of communityacquired pneumonia: a controlled before-and-after study. Clin Infect Dis 2004:39:955-63.

32. Marrie TJ, Lau CY, Wheeler SI, et al. A controlled trial of a critical pathway for treatment of community-acquired pneumonia. JAMA 2000;283:749-55.

33. Sington JD, Cottrell BJ. Analysis of the sensitivity of death certificates in 440 hospital deaths: a comparison with necropsy findings. J Clin Pathol 2002;55:499-502.

34. Covinsky KE, Palmer RM, Counsell SR, et al. Functional status before hospitalization in acutely ill older adults: validity and clinical importance of retrospective reports. J Am Geriatr Soc 2000;48:164-9.

35. Sager MA, Franke T, Inouye SK, et al. Functional outcomes of acute medical illness and hospitalization in older persons. Arch Intern Med 1996;156:645-52. 